\title{
Coordinated regulation of CB1 cannabinoid receptors and anandamide metabolism stabilize network activity during homeostatic scaling down
}

Abbreviated title: Endocannabinoids and Homeostatic Scaling-Down

Michael Ye ${ }^{1}$, Sarah K. Monroe ${ }^{1}$, Sean M. Gay², Michael L. Armstrong ${ }^{3}$, Diane E. Youngstrom ${ }^{1}$, Fabio L. Urbina1 ${ }^{1}$ Stephanie L. Gupton ${ }^{1,2}$, Nichole Reisdorph ${ }^{3}$, Graham H Diering ${ }^{1,2,4 *}$

Affiliations

1. Department of Cell Biology and Physiology, University of North Carolina at Chapel Hill, Chapel Hill, NC, USA 27599

2. UNC Neuroscience Center

3. Skaggs School of Pharmacy and Pharmaceutical Sciences, University of Colorado Anschutz Medical Campus, Aurora, CO, USA 80045

4. Carolina Institute for Developmental Disabilities

*Correspondence: graham diering@med.unc.edu

Number of pages: 36

Number of figures: 6

Number of words: abstract (241), introduction (643), discussion (1483)

Conflict of interest: The authors declare no competing financial interests

Acknowledgements: This work was supported by a grant to GHD from the National Institutes of Health (RF1AG068063), and to SLG (R01NS112326). 


\section{Abstract}

Neurons express overlapping homeostatic mechanisms to regulate synaptic function and network properties in response to perturbations of neuronal activity. Endocannabinoids (eCBs) are bioactive lipids synthesized in the post-synaptic compartments that regulate synaptic transmission, plasticity, and neuronal excitability throughout much of the brain, by activating presynaptic cannabinoid receptor CB1. The eCB system is well situated to regulate neuronal network properties and coordinate pre- and post-synaptic activity. However, the role of the eCB system in homeostatic adaptations to neuronal hyperactivity is unknown. We show that in mature cultured rat cortical neurons, chronic bicuculline treatment, known to induce homeostatic scaling-down, induces a coordinated adaptation to enhance tonic eCB signaling. Hyperexcitation triggers down regulation of fatty acid amide hydrolase (FAAH), the lipase that degrades the eCB anandamide. Subsequently, we measured an accumulation of anandamide and related metabolites, and an upregulation of total and cell surface CB1. We show that bicuculline induced downregulation of surface AMPA-type glutamate receptors and upregulation of CB1 occur through independent mechanisms. Finally, using live-cell microscopy of neurons expressing an extracellular fluorescent glutamate reporter (iGluSnFR), we confirm that cortical neurons in vitro exhibit highly synchronized network activity, reminiscent of cortical up-states in vivo. Up-state like activity in mature cortical cultures requires CB1 signaling under both control conditions and following chronic bicuculline treatment. We propose that during the adaptation to chronic neuronal hyperexcitation, tonic eCB signaling is enhanced through coordinated changes in anandamide metabolism and cell-surface CB1 expression to maintain synchronous network activity.

\section{Significance statement}

Neurons are remarkably resilient to perturbations in network activities thanks to the expression of overlapping homeostatic adaptations. In response to network hyperactivity or silencing, 
neurons can respond through changes in excitatory and inhibitory post-synaptic neurotransmitter receptors, probability of pre-synaptic neurotransmitter release, or changes in membrane excitability. The endocannabinoid system is a prominent signaling pathway at many synapses that is known to be involved in multiple forms of short and long-term synaptic plasticity. Here we find that components of the endocannabinoid system are upregulated in response to chronic hyperexcitation of cultured cortical neurons, and that endocannabinoid signaling is required to maintain synchronized network activity. This work supports a novel tonic homeostatic function for the endocannabinoid system in neurons. 


\section{Introduction}

Neurons express overlapping homeostatic mechanisms to maintain excitability and network properties in response to changes in synaptic inputs. Homeostatic scaling is a well described bidirectional phenomenon that broadly controls the strength of excitatory and inhibitory synapses through regulation of pre- and post-synaptic functions (Turrigiano, 2008;

Turrigiano et al., 1998). In response to prolonged hyperexcitation or silencing, homeostatic mechanisms are engaged in cortical and hippocampal neurons to reduce or enhance glutamatergic transmission respectively, through regulation of AMPA-type glutamate receptors (AMPARs) in the post-synapse, and regulation of synaptic vesicle release at the pre-synapse (Diering et al., 2014; O'Brien et al., 1998; Wierenga et al., 2006). GABAergic transmission is also regulated during homeostatic plasticity by pre- and post-synaptic mechanisms, generally in the opposite direction (Turrigiano, 2008). The pre- or post-synaptic locus of homeostatic mechanisms depends on the neuronal types and levels of maturation. In primary cortical and hippocampal neurons at $\sim 2$ weeks in vitro many studies have described homeostatic responses dominated by post-synaptic adaptations. With further maturation at $\sim 3$-weeks in vitro, additional pre-synaptic mechanisms are also engaged (De Gois et al., 2005; Turrigiano, 2008; Wierenga et al., 2006). Whereas many molecular mechanisms have been described underlying pre- and post-synaptic homeostatic adaptations, it is not entirely clear how pre- and post-synaptic glutamatergic and GABAergic plasticity mechanisms are coordinated to maintain stable network activity in response to network hyperexcitation.

Endocannabinoids (eCBs) are bioactive lipids that regulate neurotransmitter release and synaptic plasticity, largely through activation of pre-synaptic Cannabinoid receptor 1 (CB1) (Castillo et al., 2012). CB1 is broadly expressed in many neuron types and is known to regulate glutamatergic and GABAergic transmission, primarily through a $\mathrm{G} \alpha_{\mathrm{i}}$-coupled suppression of synaptic vesicle release (Castillo et al., 2012; Di Marzo, 2018). CB1 is also known to regulate 
cortical up-states, a prominent mode of network activity seen in isolated cortical slices or dissociated cultures in vitro, or during non-rapid eye movement (NREM) sleep in vivo (Johnson and Buonomano, 2007; Pava et al., 2014; Saberi-Moghadam et al., 2018; Steriade et al., 1993). Two major eCBs have been described: 2-arachidonyl glycerol (2-AG), and arachidonoyl ethanolamide (AEA, also called anandamide). Importantly, while both 2-AG and AEA act as agonists for CB1, these metabolites are synthesized and degraded through completely nonoverlapping sets of enzymes, suggesting that they may serve distinct functions and undergo separate regulation (Di Marzo, 2018). Unlike other neuromodulators, eCBs are not stored in vesicles for release, but are synthesized "on demand" from the catabolism of post-synaptic phospholipids in an activity-dependent manner. The eCB system constitutes an important retrograde signaling mechanism whereby activity of post-synaptic neurons can regulate presynaptic transmitter release (Castillo et al., 2012). Therefore, the eCB system is ideally situated to coordinate the activities of pre- and post-synaptic plasticity and regulate network activity during homeostatic scaling.

In the current study we have observed that in relatively mature cortical cultures, CB1 protein expression and cell-surface targeting are upregulated in response to chronic 48hr hyperexcitation caused by treatment with the $\mathrm{GABA}_{\mathrm{A}}$ receptor antagonist bicuculline. This adaptation is coordinated with a downregulation of $F A A H$ and consequent accumulation of $A E A$ and related $\mathrm{N}$-acylethanolamides (NAEs) to enhance tonic CB1-AEA signaling. We show that the well described bicuculline induced downregulation of surface AMPARs (Diering et al., 2014) is independent of CB1 signaling. Using live-cell imaging of a fluorescent glutamate neurotransmitter reporter (iGluSnFr) (Marvin et al., 2018), we observe highly synchronized glutamate release events reminiscent of synchronized action potential firing observed in dissociated cortical cultures using multi-electrode arrays, and during cortical up-states in vitro and in vivo (Pava et al., 2014; Saberi-Moghadam et al., 2018; Steriade et al., 1993). Consistent 
with a previous report (Pava et al., 2014) we show that CB1 signaling is required to maintain this cortical up-state like activity, both under control conditions and following bicuculline-induced homeostatic scaling-down. We propose that coordinated regulation of eCB metabolism and signaling maintain network activity during the response to network hyperexcitation.

\section{Materials and Methods}

\section{Primary Neuron Culture}

Primary dissociated cortical neurons were prepared from E18 Sprague-Dawley rats as previously described (Diering et al., 2014). Briefly, dissociated cortical neurons were plated onto poly-L-lysine (Sigma Aldrich) coated tissue culture dishes and glass coverslips, and fed twice a week in glial conditioned neurobasal media (Gibco) supplemented with $2 \%$ B27, $1 \%$ horse serum, 2mM GlutaMAX and 100U/mL penicillin/streptomycin (Gibco). For biochemistry experiments, neurons were plated at 600,000 cells/well into 6-well tissue culture plates (Falcon). For live-cell imaging, neurons were plated at 120,000 cells/well into 4-well chambered cover glass (Cellvis).

\section{Drug Treatment}

Drugs were prepared as $1000 x$ stocks and stored at $-20^{\circ} \mathrm{C}$. To induce homeostatic scaling, neurons were treated with $20 \mu \mathrm{M}$ bicuculline methobromide (BCC) (Tocris, $20 \mathrm{mM}$ stock in water) or $1 \mu \mathrm{M}$ tetrodotoxin citrate (TTX) (Abcam, $1 \mathrm{mM}$ stock in water) for $48 \mathrm{~h}$. In some live-cell imaging experiments, $20 \mu \mathrm{M}$ BCC or $1 \mu \mathrm{M}$ TTX was applied acutely for 10-40min. For Figure 2, neurons were treated with $20 \mu \mathrm{M} \mathrm{BCC}$ for $4,12,24$ or $48 \mathrm{~h}$ to monitor the process of homeostatic down-scaling. For Figure 3, neurons were treated for $48 \mathrm{~h}$ with a combination of $1 \mu \mathrm{M}$ MTEP

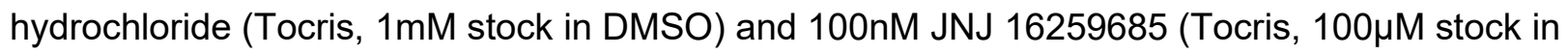
DMSO) to block mGluR1/5 signaling. For Figure 3 and 5, neurons were treated for 10-40min 
(acute application) or 48hr with 500nM AM251 (Cayman, 500 $\mu$ M stock in DMSO) to block CB1 signaling. For Figure 4, neurons were treated for 10-40min with 50 $\mu$ M D-AP5 (Cayman, 50mM stock in water) to block NMDA signaling.

\section{Antibodies}

The following mouse primary antibodies were used: anti-GluA1 (NeuroMab 75-327, 1:1,000), anti-FAAH (Abcam 1:1,000), anti-PSD95 (NeuroMab 75-028, 1:1,000,000) and anti-a-Tubulin (Abcam, 1:50,000). The following rabbit primary antibodies were used: anti-GluA1 phosphoS845 specific (Millipore 1:1,000) and anti-CB1 (Cell Signaling, 1:1,000). The following guinea pig primary antibodies were used: anti-vGluT1 (Synaptic Systems 135 304, 1:1,1000), antivGAT (Synaptic Systems 131 005, 1:1,000). The following secondary antibodies were used: goat anti-mouse 680RD (Licor, 1:10,000), goat anti-rabbit 800CW (Licor, 1:15,000) and donkey anti-guinea pig 800CW (Licor, 1:15,000).

\section{Cell surface biotinylation}

Surface biotinylation of cultured cortical neurons were performed as previously (Diering et al., 2014). Briefly, neurons were rinsed with ice-cold PBSCM (PBS containing $0.1 \mathrm{mM} \mathrm{CaCl}_{2}$ and $1 \mathrm{mM} \mathrm{MgCl} 2, \mathrm{pH} 8.0$ ), incubated in PBSCM containing $0.5 \mathrm{mg} / \mathrm{mL}$ Sulfo-NHS-SS-biotin (Thermo Scientific, $30 \mathrm{~min}, 4^{\circ} \mathrm{C}$ ), then rinsed in ice-cold PBSCM. Excess biotin was then quenched twice in PBSCM containing $20 \mathrm{mM}$ glycine $\left(2 \times 7 \mathrm{~min}, 4^{\circ} \mathrm{C}\right)$, and then the cells were washed again with ice-cold PBSCM. Cells were lysed in ice-cold lysis buffer (PBS containing 1\% Triton X-100, $0.5 \%$ sodium deoxycholate, $0.02 \%$ SDS, $1 \mathrm{mM}$ EDTA, $5 \mathrm{mM}$ sodium pyrophosphate, $1 \mu \mathrm{M}$ okadaic acid, $1 \mathrm{mM} \mathrm{Na}_{3} \mathrm{VO}_{4}$ and phosphatase inhibitor cocktail [Roche]). Lysates were precleared with centrifugation $\left(17,000 \mathrm{G}, 10 \mathrm{~min}, 4^{\circ} \mathrm{C}\right)$. Protein concentration of each pre-cleared lysate was quantified using Bradford reagent (Bio-rad), and equal amounts of proteins were incubated overnight with NeutrAvidin-coupled agarose beads (Thermo Scientific). Beads were 
washed three times with ice-cold PBS containing 1\% Triton X-100, and biotinylated proteins were eluted with $2 x \operatorname{SDS}$ sample buffer at $65^{\circ} \mathrm{C}$ for $20 \mathrm{~min}$. Cell surface or total proteins were then subjected to SDS-PAGE and analyzed by western blot.

\section{Western blot}

Lysates from cultured cortical neurons were pre-cleared with centrifugation $(17,000 \mathrm{G}, 10 \mathrm{~min}$, $\left.4^{\circ} \mathrm{C}\right)$. Protein concentration was determined using Bradford assay. Pre-cleared lysates were mixed with 2x SDS sample buffer (20\% glycerol, 100mM Tris, 5\% SDS, 5\% BME, pH 6.8) and denatured at $65^{\circ} \mathrm{C}$ for $20 \mathrm{~min}$. Equal amount of proteins were loaded and separated by SDSPAGE on hand-cast gels. Separated proteins were transferred to a nitro-cellulose membrane (GE Healthcare). Following transfer, membranes were blocked in $3 \%$ BSA in TBS for 45min at room temperature. After blocking, membranes were incubated with primary antibodies dissolved in TBST (TBS containing $0.1 \%$ Tween-20) containing 3\% BSA, overnight at $4^{\circ} \mathrm{C}$. Membranes were then washed in TBST (3x 15min) before being incubated in secondary antibodies (Licor) dissolved in TBST containing 3\% BSA and $0.01 \%$ SDS for $1 \mathrm{hr}$ at room temperature. Membranes were then washed again in TBST (3x 40min). Membranes were imaged on Licor Odyssey CLx Imaging System. Blots were analyzed and quantified using Image Studio software (Licor).

\section{Targeted mass spectrometry}

Rat primary cortical neurons were grown on $10 \mathrm{~cm}$ tissue culture plates at a density of $6 \times 10^{6}$ cells/plate for 17DIV. Culture media was aspirated, cells were rinsed with ice cold PBS and then collected and suspended by scraping in PBS containing 50nM JZL195 (Tocris) (Long et al., 2009). Cells were pelleted by centrifugation at 5,000xg for $5 \mathrm{~min}$, the supernatant was removed and cell pellets were flash frozen and stored at $-80^{\circ} \mathrm{C}$ until further analysis. Frozen cell pellets were prepared for endocannabinoid analysis as follows. Briefly, cell pellets were removed from - 
$80^{\circ} \mathrm{C}$ freezer and thawed on ice. To each sample $170 \mu$ of methanol, $20 \mu$ l of internal standard containing $200 \mathrm{ng} / \mathrm{ml}$ each of arachidonyl ethanolamide-d4 and Oleoyl ethanolamide-d4 and $2000 \mathrm{ng} / \mathrm{ml}$ of 2 -arachidonlyl glycerol-d5, and $10 \mu \mathrm{l}$ of $5 \mathrm{mg} / \mathrm{ml} \mathrm{BHT}$ in ethanol was added. The cell pellet was resuspended and then vortexed for 5 seconds. The sample was then centrifuged at $14,000 \mathrm{RPM}$ for 10 minutes at $4^{\circ} \mathrm{C}$. The supernatant was removed and then placed into a capped autosampler vial for analysis. LC/MS/MS analysis of endocannabinoids was performed as previously described (Gouveia-Figueira and Nording, 2015), with some modifications. Briefly, mass spectrometric analysis was performed on an Agilent 6490 triple quadrupole mass spectrometer in positive ionization mode. Calibration standards were analyzed over a range of concentrations from $0.025-50 p g$ on column for all of the ethanolamides and 2.5-5000pg on column for the 2-AG. The following lipids were quantified: 2-arachidonyl glycerol (2-AG), arachidonoyl ethanolamide (AEA), docosahexaenoyl ethanolamide (DHEa), docosatetraenoyl ethanolamide (DEA), linoleoyl ethanolamide (LEA), oleoyl ethanolamide (OEA), palmitoleoyl ethanolamide (POEA), palmitoyl ethanolamide (PEA), stearoyl ethanolamide (SEA). Quantitation of endocannabinoids was performed using Agilent Masshunter Quantitative Analysis software. All results were normalized to protein concentration.

\section{Transfection and Live-cell wide field microscopy}

A mixture of $1.5 \mu \mathrm{L}$ of Lipofectamine 2000 (Invitrogen) and $2.0 \mu \mathrm{g}$ of pAAV.CAG.SFiGluSnFR.A184S (Addgene plasmid \# 106198 ; gifted from Loren Looger;

\section{http://n2t.net/addgene:106198 ; RRID:Addgene_106198) in $60 \mu \mathrm{L}$ Neurobasal Medium}

(Invitrogen) was prepared and incubated at room temperature for 30 minutes. Media from neuron cultures was saved aside and cells were incubated with this mixture at $37^{\circ} \mathrm{C}$ and $5 \%$ $\mathrm{CO}_{2}$ for 30 minutes. Mixture was aspirated and replaced with original media. Time lapse wide field fluorescence videos were obtained at 5 frames per second using a Zeiss Laser Scanning Microscope (LSM) 800/Airyscan equipped with a Colibri 7 LED light source, Zeiss Axiocam 506 
CCD camera, and $63 \times / 1.4 \mathrm{NA}$ objective lens at $37^{\circ} \mathrm{C}$ and $5 \% \mathrm{CO}_{2}$. Neurons were imaged in their culture media.

\section{Live-cell Imaging Data Analysis}

Timelapse images were analyzed in FIJI by first subtracting background fluorescence measured from an empty region of the field of view then running an exponential fit bleach correction using a region of interest containing dendrites of a cell in the field of view. The dendritic arbor was traced in FIJI and designated the region of interest. Fluorescence levels in this region of interest were measured and analyzed semi-automatically with a custom code in $\mathrm{R}$, which identifies frames where synchronized fluorescent changes began by locating peaks in the second derivative of a plot of fluorescence over time. A baseline fluorescence was assigned to each synchronous event, corresponding with the fluorescence intensity value of the first frame preceding the synchronous event and change in fluorescence over background $(\Delta F / F)$ over baseline was calculated for each frame. The area under the curve of synchronous events was measured in arbitrary units, by summing the $\Delta \mathrm{F} / \mathrm{F}$ values for each frame from the initial rise from baseline to the return to baseline. Inter-event interval was calculated by counting the number of frames from the start of one event to the start of the next and converting this value to seconds based on recording frame rate. Statistics were run in Prism. For presentation purposes only, a double derivative time lapse was generated that allows for the clear visualization the change in fluorescence with time. This allows for the clear visualization of localized asynchronous glutamatergic events and global, synchronous glutamatergic events.

\section{Study design and statistical analysis}

Results are presented as mean \pm SEM from at least three independent culture preparations. Statistical significances were determined using unpaired t tests between Ctrl, BCC and TTX. One-way ANOVA and appropriate post hoc multiple comparisons were used, as indicated in 
figure legends, for comparisons of protein and targeted lipidomics at different time points in scaling down time course, for comparisons between Ctrl and BCC with or without MTEP/JNJ or AM251, and for comparisons of interevent intervals and area under curve before and after acute AM251 treatment. All statistical analyses were performed in Graphpad Prism.

\section{Results}

\section{Adaptation of the eCB system during homeostatic scaling-down}

The endocannabinoid system $(\mathrm{eCB})$ is a major neuromodulatory system that regulates neurotransmitter release at both glutamatergic and GABAergic synapses. To determine a possible role of the eCB system in homeostatic plasticity, developing (DIV11-12) and mature (DIV22-25) cultured rat cortical neurons were treated with bicuculline (BCC) or tetrodotoxin (TTX) for 48hrs, treatments previously shown to robustly induce homeostatic scaling-down and scaling-up respectively (Turrigiano et al., 1998). Neurons were surface biotinylated followed by lysis to allow for the detection of total and surface proteins (Fig. 1A). We examined the expression of AMPAR receptor subunit GluA1 and cannabinoid receptor 1 (CB1). In developing and mature cultures, BCC induced a significant downregulation in surface GluA1 and dephosphorylation of GluA1 S845 (pS845), indicating that the neurons have engaged homeostatic scaling-down, consistent with previous findings (Diering et al., 2014) (Fig. 1A-C). Network suppression with TTX caused a trend of increased surface GluA1 as expected, although these changes were not significant, and in mature cultures we observed the expected TTX-induced increase in phosphorylation of GluA1 S845 previously associated with homeostatic scaling-up (Diering et al., 2014; Kim and Ziff, 2014). In developing cultures BCC had no effect on CB1 expression, whereas TTX treatment caused a significant increase in surface, but not total, CB1 levels (Fig. 1B). In contrast, mature cultures responded to BCC by a significant 
upregulation of surface and total CB1 expression, compared to control, whereas TTX had no effect (Fig. 1C). The above data suggest that eCB system adapts to changes in network activity in a manner that depends on the maturation of the culture.

To further investigate the maturational changes in vitro, we measured synapse and endocannabinoid system protein expression in neurons at DIV8, DIV15 and DIV22 using western blot (Fig. 1D). In the second week in vitro (DIV8 vs. DIV15), we found a dramatic increase in all proteins examined (Fig. 1E). In the third week in vitro (DIV22 vs. DIV15), we found a striking increase in the expression of vesicular glutamate transporter 1 (vGluT1) and fatty acid amid hydrolase (FAAH), the principal degradative lipase for anandamide (AEA) and related $\mathrm{N}$-acylethanolamines (NAEs). Additionally, we found a moderate increase in GluA1, PSD95, and vesicular GABA transporter (vGAT) and no change in CB1 (Fig. 1E). These results suggest that, compared to developing neurons, mature neurons further undergo a significant amount of synapse maturation, especially in pre-synaptic glutamate release machinery such as vGluT1, and potentially have significantly different metabolism of NAEs as indicated by increased expression of FAAH. In our subsequent experiments we have focused on the adaptation of the eCB system to network hyperexcitability in mature neuron cultures.

\section{Temporal Dynamics of eCB Adaptations}

To further characterize the adaptations in the eCB system during homeostatic scaling down in mature cultures, we performed a time course of BCC treatment (4-48hrs) followed by western blot analysis and targeted quantification of eCB lipids using mass spectrometry. Total GluA1 showed a significant decrease beginning at $24 \mathrm{hrs}$ as expected. pS845 increased over the first 12 hours, returned to control level by $24 \mathrm{~h}$ and decreased significantly at $48 \mathrm{~h}$ (Fig. $2 \mathrm{~A}$ and B). CB1 increased significantly but only at $48 \mathrm{hr}$, suggesting that this was a delayed response (Fig. 2B). Interestingly, FAAH decreased progressively starting at $12 \mathrm{hr}$ and remained significantly downregulated through the $48 \mathrm{hr}$ time course, suggesting that adaptations in eCB metabolism 
are an earlier step in homeostatic downscaling (Fig. 2B). Accordingly, targeted mass spectrometry of eCBs showed that while 2-AG upregulation was a prominent feature of BCC treatment at $4 \mathrm{hr}$, a significant upregulation of AEA became the dominant response at $24 \mathrm{hr}$ and onward. By 48hr, multiple NAEs, all FAAH substrates, were significantly upregulated (Fig. 2C). These findings suggest that during the induction and expression of homeostatic scaling-down there is a stepwise adaptation in the eCB system starting with downregulation of FAAH protein and consequent increases in the abundance of AEA and other NAEs, followed by the delayed upregulation of CB1. Changes in AEA metabolism and upregulation of CB1 likely synergize to enhance tonic eCB signaling.

\section{Coordination of presynaptic CB1 signaling and postsynaptic glutamatergic signaling}

Homeostatic scaling-down in cultured rodent neurons is primarily understood as a postsynaptic adaptation that involves downregulation of synaptic AMPARs. Since BCC-induced up-regulation of CB1 receptors suggests a coordinated pre- and post-synaptic response, we next performed a series of pharmacology experiments to examine the relationship between post-synaptic glutamatergic signaling and pre-synaptic cannabinergic signaling during BCC-induced scalingdown. A previous report has shown that hyperexcitation caused by BCC resulted in activation of type I mGluR1/5 and that a cocktail of non-competitive mGluR1/5 inhibitors could prevent postsynaptic scaling-down of AMPARs (Hu et al., 2010). We confirmed this finding by treating neurons with a cocktail of inhibitors $1 \mu \mathrm{M}$ MTEP and 100nM JNJ16259685 (MTEP/JNJ) and showing that this treatment prevented BCC-induced downregulation of surface GluA1 and partially blocked the dephosphorylation of pS845 (Fig. 3B). However, MTEP/JNJ treatment had no effect on BCC-induced upregulation of total and surface CB1 (Fig. 3B). This result suggests that pre-synaptic adaptation of CB1 during scaling-down is independent of post-synaptic glutamatergic signaling. 
Next, we blocked cannabinergic signaling in cultured rat cortical neurons by treating with selective CB1 antagonist AM251 (500nM) and examined the effect on post-synaptic adaptation during scaling-down. AM251 treatment had no effect on surface GluA1 and pS845 and did not prevent the BCC-induced downregulation of surface GluA1 and pS845 (Fig. 3D). Interestingly, the BCC-induced upregulation of CB1 was not prevented by AM251, suggesting that CB1 upregulation is also independent of CB1 signaling. Together, these findings suggest that neurons respond to network hyperexcitability with coordinated cannabinergic and glutamatergic adaptations, which occur through independent molecular mechanisms.

\section{CB1 signaling and excitatory network activity}

CB1 signaling is well characterized to regulate pre-synaptic vesicle release dynamics. CB1 signaling is also involved in the maintenance of excitatory up-states, a prominent mode of network activity in cortical circuits, especially during NREM sleep (Pava et al., 2014; Steriade et al., 1993). Since up-state like synchronous activity is also well documented in organotypic slices (Johnson and Buonomano, 2007; Sanchez-Vives and McCormick, 2000) and cultured cortical neurons (Canepari et al., 1997; Kamioka et al., 1996b; Saberi-Moghadam et al., 2018), we visualized excitatory network activity in culture by transfecting rat cortical neurons with the highaffinity variant of fluorescent glutamate biosensor iGluSnFR-A184S (Marvin et al., 2013; Marvin et al., 2018). iGluSNFR has a baseline fluorescence and is targeted to the extracellular plasma membrane by fusion with the PDGFR $\beta$ transmembrane domain allowing for the visualization of dendrites and dendritic spines. Glutamatergic activity is reflected by a transient increase in iGluSNFR fluorescence $(\Delta F / F)$.

Neurons were transfected with iGluSNFR at DIV17-18 and imaged at DIV21-24 for 60s at $5 \mathrm{~Hz}$ by wide-field fluorescence microscopy. We observed two drastically different patterns of glutamatergic events: highly synchronous events in which fluorescence across the entire dendritic arbor was increased (Fig. 4A), and asynchronous events highly localized to dendritic 
spines or single dendritic segments (Fig. 4B). Under control conditions, the synchronous events were recurring and regularly spaced, showed a rapid rise time to a single peak, followed by a decay within 10-15s. Amplitudes of synchronous events vary between neurons, possibly reflecting cell to cell differences in integration within the excitatory network (examples shown in Fig. 4C). We speculate that the larger synchronous glutamate events detected with iGluSNFR reflect cortical up-state like activity that has been readily described in cortical cultures using multi-electrode array (MEA) recordings (Saberi-Moghadam et al., 2018). Published results using MEA recording show that cortical up-state activity is strongly inhibited by $\mathrm{N}$-methyl-D-aspartate receptor (NMDAR) antagonist D-AP5, and completely abolished by voltage-gated sodium channel inhibitor TTX (Canepari et al., 1997; Fong et al., 2015; Kamioka et al., 1996b; Robinson et al., 1993). Accordingly, with iGluSNFR imaging we observed that synchronous glutamatergic events were dramatically reduced in amplitude by acute D-AP5 and completely suppressed by TTX (Fig. 4D and E). In contrast, the smaller asynchronous events persisted in acute TTX application (data not shown), suggesting that they largely correspond to mini excitatory postsynaptic currents (mEPSCs) typically observed in patch-clamp recordings (Turrigiano et al., 1998). Such spontaneous network activities in vitro are rapidly converted to a burst firing pattern by $\mathrm{GABA}_{\mathrm{A}}$ receptor antagonism (Bateup et al., 2013; Odawara et al., 2016). Accordingly, we observed that acute BCC treatment resulted in a plateau in iGluSNFR $\triangle F / F$ during the peak followed by a rapid decline in fluorescence intensity, suggesting BCC results in a transiently sustained glutamate release during synchronous events (Fig. 4F). Following 48h chronic BCC treatment, the plateau of synchronous events was absent, and the frequency and amplitude of synchronous events were comparable to control level (Fig. 5A and B). Similar return from burst firing has been documented using MEA recording and largely match our observed time course (Bateup et al., 2013; Kaufman et al., 2014; Saberi-Moghadam et al., 2018). Together, our observations of synchronous glutamatergic events with iGluSNFR and their modulation by D- 
AP5, TTX, and BCC, suggest that these events are analogous to up-state activity described in cortical cultures and slices in vitro, and during NREM sleep in vivo.

To test the role of CB1 signaling in synchronized glutamatergic network activity, and in the homeostatic adaptation to network hyperexcitability, we acutely blocked CB1 with AM251 under control conditions or following chronic BCC 48h treatment. Under both control and chronic BCC conditions, acute AM251 significantly suppressed the magnitude of synchronous glutamate release events, as measured by decreased area under the curve (Fig. 5A and B). This observation suggests that CB1 signaling is required to maintain cortical up-state like activity, consistent with a previous report (Pava et al., 2014). Further, under control conditions CB1 inhibition reduced the frequency of glutamatergic events (Fig. 5A and B), whereas, following chronic BCC, AM251 application caused glutamatergic events of low amplitude to become more frequent (Fig. 5A and $B$ ). These observations suggest that $C B 1$ signaling supports synchronous glutamatergic network activity under control conditions and during homeostatic adaptation to hyperexcitation.

\section{Discussion}

The eCB system has been well described to participate in multiple forms of short and long term synaptic plasticity (Castillo et al., 2012). For example, CB1 signaling underlies the phenomena of depolarization induced suppression of excitation/inhibition (DSE and DSI). However, to our knowledge, homeostatic adaptations of the eCB system in vitro have only been studied in the context of chronic network silence (Kim and Alger, 2010; Song et al., 2015). In this study we provide quantitative analysis of the adaptations of the eCB system to network hyperexcitability induced by chronic BCC treatment in cultured rat cortical neurons, an in vitro system that has been used to investigate the molecular mechanisms of homeostatic scaling-down (Diering et al., 
2014; Hu et al., 2010; Turrigiano et al., 1998). We report evidence of rapid and persistent downregulation of FAAH, consequent upregulation of bioactive NAEs including AEA, and delayed upregulation of CB1. Furthermore, we show that pre-synaptic cannabinergic adaptations and post-synaptic glutamatergic adaptations occur through independent mechanisms. Finally, we show that endocannabinoid signaling through CB1 is important for maintaining synchronized glutamatergic network activity following the expression of homeostatic downscaling (Fig. 6).

In developing cultures $\sim 2$ weeks in vitro, homeostatic plasticity predominantly reflects changes in post-synaptic receptor density (Diering et al., 2014; Turrigiano, 2008). Additional pre-synaptic homeostatic adaptations are engaged as neurons mature in culture $\sim 3$ weeks in vitro (Wierenga et al., 2006). Similarly, our data show that the more mature 3-week-old neuron cultures respond to chronic hyperexcitation in part through upregulation of surface and total CB1 (Fig.1). Other adaptations in mature cultures include homeostatic regulation of vesicular transporters (De Gois et al., 2005). Compared to developing cultures, mature cultures undergo further synaptic, dendritic, and functional development, which may confer greater need to regulate pre-synaptic components and network activities during homeostatic scaling-down (Harrill et al., 2015; Wagenaar et al., 2006). Mature cultures show more developed synapses as evidenced by increased expression of post-synaptic ion channel GluA1 and pre-synaptic vesicle release machineries vGluT1 and vGAT (Fig.1E). Although total CB1 levels were similar at DIV15 and DIV21, we observed a clear increase in the expression of FAAH, indicating that the eCB system is still undergoing maturation. We suggest that the upregulation of $F A A H$, and the emergence of the BCC-induced upregulation of $\mathrm{CB} 1$ during the maturation of cortical neurons in vitro, correlates with the increase in synchronous network activity associated with neuronal maturation in vitro (Kamioka et al., 1996a).

\section{2-AG versus $A E A$}


2-AG and AEA are both well described agonists of the CB1 receptor. However, these two metabolites have completely non-overlapping enzymes that mediate synthesis and degradation, suggesting that the two most prominent eCBs undergo distinct regulation and serve separate physiological functions (Di Marzo, 2018). 2-AG mediates major forms of eCB-dependent plasticity, such as DSI, DSE and mGluR-dependent LTD (Castillo et al., 2012; Tanimura et al., 2010; Yoshino et al., 2011). However, AEA has been implicated in cortical up-sate activity, sleep stability and memory (Busquets-Garcia et al., 2011; Murillo-Rodriguez et al., 1998; Pava et al., 2014). Our results indicate that AEA but not 2-AG exerts tonic action during homeostatic scaling-down (Fig. 2). Some properties make AEA more suitable for tonic signaling. First, AEA is considered a partial agonist at CB1, which makes it less efficacious than full agonists such as 2-AG (Burkey et al., 1997; Glass and Northup, 1999; Sugiura et al., 1996). By regulating AEA during global downscaling of synaptic strength, neurons may preserve sensitivity to 2-AGmediated "fine-tuning" of individual synapses. Second, sustained 2-AG signaling results in desensitization of CB1, whereas sustained AEA signaling does not (Schlosburg et al., 2010). By minimizing receptor desensitization, neurons may maintain tonic endocannabinoid signaling throughout the relatively long time-frame of homeostatic plasticity and avoid functional antagonism due to sustained 2-AG signaling, while at the same time preserving the short term plasticity mediated by 2-AG. Indeed, Kim and Alger (2010) showed that chronic inactivity in hippocampal slices reduces endocannabinoid tone by regulating AEA degradation instead of 2AG. Thus, we hypothesize that 2-AG and AEA indeed mediate distinct mechanisms: a phasic action of 2-AG mediating short term plasticity, and a tonic action of AEA that maintains neuronal network activity.

Our time course data suggest that multiple FAAH substrates have become significantly upregulated by $48 \mathrm{~h}$ (Fig. 2C). Since FAAH is the major enzyme for catabolizing multiple endocannabinoid substrates in the NAE family, it is possible that cellular levels of NAEs are 
regulated through a common mechanism to mediate coordinated signaling events (Di Marzo, 2018). Two such NAEs, oleoylethanolamide (OEA) and palmitoylethanolamide (PEA), are agonists for nuclear endocannabinoid receptor PPARa, and have been implicated in sleep-wake regulation and memory acquisition (Mazzola et al., 2009; Murillo-Rodriguez et al., 2011). Indeed, PPAR $\alpha$ has been described to be required for expression of the immediate early gene Arc in response to hippocampal neuronal activity through CREB-mediated transcriptional control of plasticity-related molecules (Roy et al., 2013). Thus, we speculate that AEA-CB1 signaling is only one part of a coordinated neuronal response that synergizes with signaling pathways activated by other bioactive NAEs. A possible role for OEA, PEA and/or PPARa should be investigated in future studies.

CB1, cortical up-states and NREM sleep

During NREM sleep, cortical networks alter between periods of coordinated firing (up-state) and periods of network silence (down-state) (Steriade et al., 1993). Similarly, dissociated culture engage in a default sleep-like state where the network fires in synchronized bursts reminiscent of cortical up-states (Canepari et al., 1997; Kamioka et al., 1996b; Maeda et al., 1995; Robinson et al., 1993; Saberi-Moghadam et al., 2018). Tonic CB1 signaling has been shown to support up-state activity in organotypic slices in vitro, and NREM sleep stability in vivo (Kesner and Lovinger, 2020; Pava et al., 2014). We show here that tonic CB1 signaling is similarly required to support NREM like up-states in dissociated cortical cultures. We suggest that, following network hyperexcitation, enhancement of cannabinergic tone is required for the maintenance of synchronous network activity, although the reasons for this, or the mechanism by which CB1 supports network activity, is not known (Fig. 6).

CB1 regulates synaptic transmission through a Gai mediated suppression of vesicle release, but also controls axonal excitability through the regulation of voltage-gated $\mathrm{Ca}^{++}$and $\mathrm{K}^{+}$ channels (Di Marzo, 2018; Guo and Ikeda, 2004). In previous work it was speculated that CB1 
supports up-states by shifting the balance of excitation and inhibition through relative differences in the suppression of glutamate and GABA release (Pava et al., 2014). However, we observed that CB1 is required for synchronized up-state like activity even in the presence of BCC, implying that CB1 supports up-states independent of GABAergic transmission (Fig. 5). We speculate that CB1 signaling promotes up-state activity through the regulation of axonal excitability (Fig. 6). A candidate mechanism is the activation of G protein-coupled inwardlyrectifying potassium channels (GIRKs), that are activated by $C B 1$ and have recently been implicated in NREM sleep (Guo and Ikeda, 2004; Zou et al., 2019). In addition to reducing the magnitude of up-state like events, CB1 inhibition significantly altered the frequency of the remaining smaller glutamatergic events, but in the opposite direction under control and chronic BCC conditions. We speculate these differential effects on event frequency are due to disinhibition of synaptic vesicle release, that occurs at both glutamatergic and GABAergic synapses. Under control conditions, CB1 inhibition reduced the frequency of glutamatergic events, possibly through a greater disinhibition of GABAergic synapses, favoring network suppression. In the presence of BCC GABAergic transmission is absent, and consequently, AM251 increased the frequency of small glutamatergic events through disinhibition of glutamate release.

Certain molecular mechanisms engaged during homeostatic scaling-down in cortical cultures, including changes in AMPAR phosphorylation and Homer1a-dependent activation of mGluR1/5 (Diering et al., 2014; Hu et al., 2010), have also been observed to occur during sleep in vivo (Diering et al., 2017). This is in accordance with a prominent hypothesis that the restorative benefits of sleep are mediated in part by a broad but selective scaling-down of excitatory synapses (de Vivo et al., 2017; Tononi and Cirelli, 2014). Here we show that FAAH, $\mathrm{AEA}$, and $\mathrm{CB} 1$ are each regulated during scaling-down to promote tonic cannabinergic signaling. In a previous proteomics study we found that FAAH is downregulated at forebrain 
synapses during sleep in vivo (Diering et al., 2017). Accordingly, we recently found that synaptic AEA and related NAEs are upregulated during sleep in adult mice, and that activation of CB1 increases sleep time and stability, whereas inhibition of CB1 suppresses sleep and drives sleep fragmentation (this work is available as a pre-print on BioRxiv https://www.biorxiv.org/content/10.1101/2021.05.10.443432v1.article-info). These findings support a growing body of literature that eCB signaling promotes sleep, particularly by promoting the stability of NREM sleep (Bogathy et al., 2019; Kesner and Lovinger, 2020; Pava et al., 2016). Our current findings provide an additional parallel between homeostatic scalingdown in culture, and the synaptic changes that occur during sleep, and suggest that stabilization of synchronous cortical activity provides the basis for the NREM promoting effects of eCB signaling.

\section{References}

Bateup, H.S., Denefrio, C.L., Johnson, C.A., Saulnier, J.L., and Sabatini, B.L. (2013). Temporal dynamics of a homeostatic pathway controlling neural network activity. Front Mol Neurosci 6 , 28.

Bogathy, E., Papp, N., Vas, S., Bagdy, G., and Tothfalusi, L. (2019). AM-251, A Cannabinoid Antagonist, Modifies the Dynamics of Sleep-Wake Cycles in Rats. Front Pharmacol 10, 831. Burkey, T.H., Quock, R.M., Consroe, P., Ehlert, F.J., Hosohata, Y., Roeske, W.R., and Yamamura, H.I. (1997). Relative efficacies of cannabinoid CB1 receptor agonists in the mouse brain. European Journal of Pharmacology 336, 295-298.

Busquets-Garcia, A., Puighermanal, E., Pastor, A., de la Torre, R., Maldonado, R., and Ozaita, A. (2011). Differential role of anandamide and 2-arachidonoylglycerol in memory and anxietylike responses. Biol Psychiatry 70, 479-486. 
Canepari, M., Bove, M., Maeda, E., Cappello, M., and Kawana, A. (1997). Experimental analysis of neuronal dynamics in cultured cortical networks and transitions between different patterns of activity. Biol Cybern 77, 153-162.

Castillo, P.E., Younts, T.J., Chavez, A.E., and Hashimotodani, Y. (2012). Endocannabinoid signaling and synaptic function. Neuron $76,70-81$.

De Gois, S., Schafer, M.K., Defamie, N., Chen, C., Ricci, A., Weihe, E., Varoqui, H., and Erickson, J.D. (2005). Homeostatic scaling of vesicular glutamate and GABA transporter expression in rat neocortical circuits. J Neurosci 25, 7121-7133.

de Vivo, L., Bellesi, M., Marshall, W., Bushong, E.A., Ellisman, M.H., Tononi, G., and Cirelli, C. (2017). Ultrastructural evidence for synaptic scaling across the wake/sleep cycle. Science 355, 507-510.

Di Marzo, V. (2018). New approaches and challenges to targeting the endocannabinoid system. Nat Rev Drug Discov 17, 623-639.

Diering, G.H., Gustina, A.S., and Huganir, R.L. (2014). PKA-GluA1 coupling via AKAP5 controls AMPA receptor phosphorylation and cell-surface targeting during bidirectional homeostatic plasticity. Neuron $84,790-805$.

Diering, G.H., Nirujogi, R.S., Roth, R.H., Worley, P.F., Pandey, A., and Huganir, R.L. (2017). Homer1a drives homeostatic scaling-down of excitatory synapses during sleep. Science 355, $511-515$.

Fong, M.F., Newman, J.P., Potter, S.M., and Wenner, P. (2015). Upward synaptic scaling is dependent on neurotransmission rather than spiking. Nat Commun 6, 6339.

Glass, M., and Northup, J.K. (1999). Agonist selective regulation of G proteins by cannabinoid $\mathrm{CB}(1)$ and $\mathrm{CB}(2)$ receptors. Mol Pharmacol 56, 1362-1369.

Gouveia-Figueira, S., and Nording, M.L. (2015). Validation of a tandem mass spectrometry method using combined extraction of 37 oxylipins and 14 endocannabinoid-related compounds including prostamides from biological matrices. Prostaglandins Other Lipid Mediat 121, 110-121. 
Guo, J., and Ikeda, S.R. (2004). Endocannabinoids modulate N-type calcium channels and Gprotein-coupled inwardly rectifying potassium channels via CB1 cannabinoid receptors heterologously expressed in mammalian neurons. Mol Pharmacol 65, 665-674.

Harrill, J.A., Chen, H., Streifel, K.M., Yang, D., Mundy, W.R., and Lein, P.J. (2015). Ontogeny of biochemical, morphological and functional parameters of synaptogenesis in primary cultures of rat hippocampal and cortical neurons. Mol Brain 8, 10.

Hu, J.H., Park, J.M., Park, S., Xiao, B., Dehoff, M.H., Kim, S., Hayashi, T., Schwarz, M.K., Huganir, R.L., Seeburg, P.H., et al. (2010). Homeostatic scaling requires group I mGluR activation mediated by Homer1a. Neuron 68, 1128-1142.

Johnson, H.A., and Buonomano, D.V. (2007). Development and plasticity of spontaneous activity and Up states in cortical organotypic slices. J Neurosci 27, 5915-5925.

Kamioka, H., Maeda, E., Jimbo, Y., Robinson, H.P., and Kawana, A. (1996a). Spontaneous periodic synchronized bursting during formation of mature patterns of connections in cortical cultures. Neurosci Lett 206, 109-112.

Kamioka, H., Maeda, E., Jimbo, Y., Robinson, H.P.C., and Kawana, A. (1996b). Spontaneous periodic synchronized bursting during formation of mature patterns of connections in cortical cultures. Neuroscience Letters 206, 109-112.

Kaufman, M., Reinartz, S., and Ziv, N.E. (2014). Adaptation to prolonged neuromodulation in cortical cultures: an invariable return to network synchrony. BMC Biol 12, 83.

Kesner, A.J., and Lovinger, D.M. (2020). Cannabinoids, Endocannabinoids and Sleep. Front Mol Neurosci 13, 125.

Kim, J., and Alger, B.E. (2010). Reduction in endocannabinoid tone is a homeostatic mechanism for specific inhibitory synapses. Nat Neurosci 13, 592-600.

Kim, S., and Ziff, E.B. (2014). Calcineurin mediates synaptic scaling via synaptic trafficking of Ca2+-permeable AMPA receptors. PLoS Biol 12, e1001900. 
Long, J.Z., Nomura, D.K., Vann, R.E., Walentiny, D.M., Booker, L., Jin, X., Burston, J.J., SimSelley, L.J., Lichtman, A.H., Wiley, J.L., et al. (2009). Dual blockade of FAAH and MAGL identifies behavioral processes regulated by endocannabinoid crosstalk in vivo. Proc Natl Acad Sci U S A 106, 20270-20275.

Maeda, E., Robinson, H.P., and Kawana, A. (1995). The mechanisms of generation and propagation of synchronized bursting in developing networks of cortical neurons. The Journal of Neuroscience 15, 6834-6845.

Marvin, J.S., Borghuis, B.G., Tian, L., Cichon, J., Harnett, M.T., Akerboom, J., Gordus, A., Renninger, S.L., Chen, T.W., Bargmann, C.I., et al. (2013). An optimized fluorescent probe for visualizing glutamate neurotransmission. Nat Methods 10, 162-170.

Marvin, J.S., Scholl, B., Wilson, D.E., Podgorski, K., Kazemipour, A., Muller, J.A., Schoch, S., Quiroz, F.J.U., Rebola, N., Bao, H., et al. (2018). Stability, affinity, and chromatic variants of the glutamate sensor iGluSnFR. Nat Methods 15, 936-939.

Mazzola, C., Medalie, J., Scherma, M., Panlilio, L.V., Solinas, M., Tanda, G., Drago, F., Cadet, J.L., Goldberg, S.R., and Yasar, S. (2009). Fatty acid amide hydrolase (FAAH) inhibition enhances memory acquisition through activation of PPAR-alpha nuclear receptors. Learn Mem $16,332-337$.

Murillo-Rodriguez, E., Palomero-Rivero, M., Millan-Aldaco, D., Arias-Carrion, O., and DruckerColin, R. (2011). Administration of URB597, oleoylethanolamide or palmitoylethanolamide increases waking and dopamine in rats. PLoS One 6, e20766.

Murillo-Rodriguez, E., Sanchez-Alavez, M., Navarro, L., Martinez-Gonzalez, D., Drucker-Colin, R., and Prospero-Garcia, O. (1998). Anandamide modulates sleep and memory in rats. Brain Res 812, 270-274.

O'Brien, R.J., Kamboj, S., Ehlers, M.D., Rosen, K.R., Fischbach, G.D., and Huganir, R.L. (1998). Activity-dependent modulation of synaptic AMPA receptor accumulation. Neuron 21, 1067-1078. 
Odawara, A., Katoh, H., Matsuda, N., and Suzuki, I. (2016). Physiological maturation and drug responses of human induced pluripotent stem cell-derived cortical neuronal networks in longterm culture. Sci Rep 6, 26181.

Pava, M.J., den Hartog, C.R., Blanco-Centurion, C., Shiromani, P.J., and Woodward, J.J. (2014). Endocannabinoid modulation of cortical up-states and NREM sleep. PLoS One 9, e88672.

Pava, M.J., Makriyannis, A., and Lovinger, D.M. (2016). Endocannabinoid Signaling Regulates Sleep Stability. PLoS One 11, e0152473.

Robinson, H.P., Kawahara, M., Jimbo, Y., Torimitsu, K., Kuroda, Y., and Kawana, A. (1993). Periodic synchronized bursting and intracellular calcium transients elicited by low magnesium in cultured cortical neurons. J Neurophysiol 70, 1606-1616.

Roy, A., Jana, M., Corbett, G.T., Ramaswamy, S., Kordower, J.H., Gonzalez, F.J., and Pahan, K. (2013). Regulation of cyclic AMP response element binding and hippocampal plasticityrelated genes by peroxisome proliferator-activated receptor alpha. Cell Rep 4, 724-737. Saberi-Moghadam, S., Simi, A., Setareh, H., Mikhail, C., and Tafti, M. (2018). In vitro Cortical Network Firing is Homeostatically Regulated: A Model for Sleep Regulation. Sci Rep 8, 6297. Sanchez-Vives, M.V., and McCormick, D.A. (2000). Cellular and network mechanisms of rhythmic recurrent activity in neocortex. Nat Neurosci 3, 1027-1034.

Schlosburg, J.E., Blankman, J.L., Long, J.Z., Nomura, D.K., Pan, B., Kinsey, S.G., Nguyen, P.T., Ramesh, D., Booker, L., Burston, J.J., et al. (2010). Chronic monoacylglycerol lipase blockade causes functional antagonism of the endocannabinoid system. Nat Neurosci 13, 11131119.

Song, Y., Zhang, J., and Chen, C. (2015). Fine-tuning of synaptic upscaling at excitatory synapses by endocannabinoid signaling is mediated via the CB1 receptor. Sci Rep 5, 16257. 
Steriade, M., Nunez, A., and Amzica, F. (1993). Intracellular analysis of relations between the slow $(<1 \mathrm{~Hz})$ neocortical oscillation and other sleep rhythms of the electroencephalogram. The Journal of Neuroscience 13, 3266-3283.

Sugiura, T., Kodaka, T., Kondo, S., Tonegawa, T., Nakane, S., Kishimoto, S., Yamashita, A., and Waku, K. (1996). 2-Arachidonoylglycerol, a putative endogenous cannabinoid receptor ligand, induces rapid, transient elevation of intracellular free $\mathrm{Ca} 2+$ in neuroblastoma $\mathrm{x}$ glioma hybrid NG108-15 cells. Biochem Biophys Res Commun 229, 58-64.

Tanimura, A., Yamazaki, M., Hashimotodani, Y., Uchigashima, M., Kawata, S., Abe, M., Kita, Y., Hashimoto, K., Shimizu, T., Watanabe, M., et al. (2010). The endocannabinoid 2arachidonoylglycerol produced by diacylglycerol lipase alpha mediates retrograde suppression of synaptic transmission. Neuron $65,320-327$.

Tononi, G., and Cirelli, C. (2014). Sleep and the price of plasticity: from synaptic and cellular homeostasis to memory consolidation and integration. Neuron $81,12-34$.

Turrigiano, G.G. (2008). The self-tuning neuron: synaptic scaling of excitatory synapses. Cell $135,422-435$.

Turrigiano, G.G., Leslie, K.R., Desai, N.S., Rutherford, L.C., and Nelson, S.B. (1998). Activitydependent scaling of quantal amplitude in neocortical neurons. Nature 391, 892-896.

Wagenaar, D.A., Pine, J., and Potter, S.M. (2006). An extremely rich repertoire of bursting patterns during the development of cortical cultures. BMC Neurosci 7, 11.

Wierenga, C.J., Walsh, M.F., and Turrigiano, G.G. (2006). Temporal regulation of the expression locus of homeostatic plasticity. J Neurophysiol 96, 2127-2133.

Yoshino, H., Miyamae, T., Hansen, G., Zambrowicz, B., Flynn, M., Pedicord, D., Blat, Y., Westphal, R.S., Zaczek, R., Lewis, D.A., et al. (2011). Postsynaptic diacylglycerol lipase mediates retrograde endocannabinoid suppression of inhibition in mouse prefrontal cortex. J Physiol 589, 4857-4884. 
Zou, B., Cao, W.S., Guan, Z., Xiao, K., Pascual, C., Xie, J., Zhang, J., Xie, J., Kayser, F., Lindsley, C.W., et al. (2019). Direct activation of G-protein-gated inward rectifying K+ channels promotes nonrapid eye movement sleep. Sleep 42. 


\section{Figure Legends}

Figure $1 \mathrm{CB} 1$ expression is upregulated during homeostatic downscaling in mature neurons.

(A) Representative western blots of surface and total protein expression in developing (DIV11-

12) and mature (DIV21-25) cultured cortical neurons treated for $48 \mathrm{~h}$ with control media (Ctrl),

$20 \mu \mathrm{M}$ bicuculline $(\mathrm{BCC})$ or $1 \mu \mathrm{M}$ tetrodotoxin (TTX). (B,C) Quantification of data shown in (A) in developing neurons (B) and mature neurons (C). BCC and TTX treated samples are normalized to control treatment. BCC induced downregulation surface GluA1 and S845 dephosphorylation regardless of age, and upregulation of total and surface CB1 only in mature neurons. (D,E) Representative western blots and quantification of total protein expression in cultured cortical neurons at DIV8, DIV15 and DIV22. Results are normalized for each protein to its expression levels at DIV15, and presented as mean \pm SEM from at least three independent culture preparations with quadruplicate wells. Standard unpaired t-test was used for $(\mathbf{B}, \mathbf{C})$ and one-way ANOVA with Dunnett's multiple comparison test was used for (E). ${ }^{*} p \leq 0.05,{ }^{* *} p \leq 0.01,{ }^{* *} p \leq$ $0.001,{ }^{* * \star *} p \leq 0.0001$

Figure 2 Time course of homeostatic down-scaling in rat primary cortical neuron culture. (A,B) Representative western blots and quantification of total protein expression in DIV17 neurons treated by control media for $48 \mathrm{~h}$ (Ctrl $48 \mathrm{~h}$ ) or by $20 \mu \mathrm{M}$ bicuculline (BCC) for $4 \mathrm{~h}, 12 \mathrm{~h}, 24 \mathrm{~h}$ or $48 \mathrm{~h}$. FAAH is significantly downregulated from $12 \mathrm{~h}$ and onward, but CB1 do not become significantly upregulated until the end of the $48 \mathrm{~h}$ time course. Results are normalized for each protein to its expression levels in vehicle $48 \mathrm{~h}$ (dotted line), and presented as mean \pm SEM from four to six independent culture preparations with duplicate wells. (C) Quantification of targeted lipidomics analyzing 2-AG, AEA and various n-acylethanolamines (NAEs) in DIV17 neurons untreated or treated by $20 \mu \mathrm{M} \mathrm{BCC}$ for $4 \mathrm{~h}, 24 \mathrm{~h}$ or $48 \mathrm{~h}$. Note that the neurons transition from a $2-\mathrm{AG}$ dominated response at $4 \mathrm{~h}$ to an AEA- and NAEs-dominated response at $24 \mathrm{~h}$ and $48 \mathrm{~h}$, corresponding with the downregulation of FAAH shown in (B). Results from each treatment 
group are normalized to a matching control, and presented as mean \pm SEM from three to four independent culture preparations with duplicate plates. One-way ANOVA with Dunnett's multiple comparison test ${ }^{*} p \leq 0.05,{ }^{* *} p \leq 0.01,{ }^{* * *} p \leq 0.001,{ }^{* * * *} p \leq 0.0001$.

Figure 3 Glutamatergic and cannabinergic adaptations occur through independent mechanisms. (A,B) Representative blots and quantifications of surface and total protein

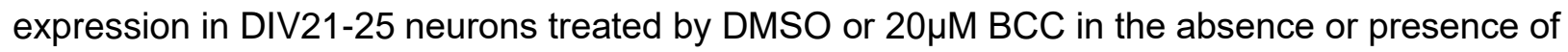
$1 \mu \mathrm{M}$ MTEP hydrochloride, a selective mGluR5 antagonist, and 100nM JNJ 16259685, a selective mGluR1 antagonist (MTEP/JNJ). MTEP/JNJ blocked BCC-induced downregulation of surface GluA1 but not the upregulation of surface CB1. (C,D) Representative blots and quantifications of surface and total protein expression in DIV21-25 neurons treated by DMSO or $20 \mu \mathrm{M}$ bicuculline $(\mathrm{BCC})$ in the absence or presence of 500nM AM251, a selective CB1 antagonist. AM251 did not block BCC-induced downregulation of surface GluA1 or the upregulation of surface CB1. Results are normalized for each protein to its expression levels in control group, and presented as mean \pm SEM from four to six independent culture preparations with triplicate wells. One-way ANOVA with Šidák's multiple comparison test ${ }^{*} p \leq 0.05,{ }^{* *} p \leq$ $0.01,{ }^{* * *} p \leq 0.001,{ }^{* * *} p \leq 0.0001$.

Figure 4 Detection of synchronous and asynchronous release of glutamate by iGluSNFR in mature (DIV21-25) neurons (A) Double derivative images showing spontaneous changes in GluSnFR fluorescence corresponding to a single synchronous glutamate event under control condition. (B) Double derivative images showing spontaneous changes in iGluSNFR fluorescence corresponding to multiple asynchronous glutamate events. Localized events are indicated by yellow arrows. (C-F) Representative traces of iGluSNFR time-lapse recorded in DIV21-25 cultured cortical neurons untreated (Ctrl, C) or treated with acute application of $50 \mu \mathrm{M}$

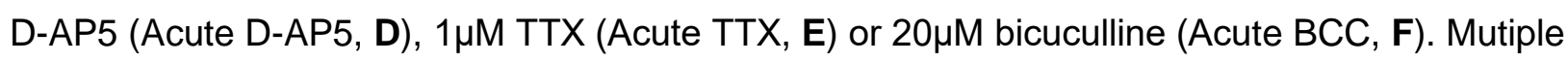
example traces are shown each representing a separate transfected neuron. While acute BCC 
causes a plateau in the synchronous events, acute D-AP5 leads to dramatically reduced amplitude of synchronous events and TTX completely abolishes them. Results are presented as fluorescence for each recording normalized to its minimum fluorescence ( $F / F_{\text {min }}$, dotted line). Scale bar represents 10 s (horizontal) and 10\% change from minimum fluorescence (vertical). Ctrl data in panel $(\mathbf{C})$ is from the same set of data as in Figure $\mathbf{5 .}$

Figure 5 Acute application of AM251 blocks synchronous up-state like glutamatatergic events (A) Representative traces of GluSnFR time-lapse recorded in DIV21-25 neurons untreated (Ctrl) or treated with bicuculline for 48hr (BCC 48hr) followed by acute application (10-40min) of AM251 (Acute AM251 and BCC 48hr + Acute AM251). Results are presented as fluorescence for each recording normalized to its minimum fluorescence $\left(F / F_{\min }\right.$, dotted line). Scale bar represents 10 s (horizontal) and 10\% change from minimum fluorescence (vertical). (B) Quantification of interevent intervals (left) and area under curve (right) of data shown in (A). AM251 causes synchronous glutamate events to become fragmented, even in the presence of BCC. Results are presented as mean \pm SEM from three to six independent culture preparations with four to eight wells in each culture. One-way ANOVA with Šidák's multiple comparison test. ${ }^{*} p \leq 0.05,{ }^{* *} p \leq 0.01,{ }^{* * *} p \leq 0.001,{ }^{* * * *} p \leq 0.0001$.

Figure 6 Model of homeostatic adaptation of the endocannabinoid system during scaling-down. BCC induced downscaling drives coordinated remodeling of synapses: 1. Down regulation of surface AMPARs, 2. Down regulation of FAAH, 3. Accumulation of FAAH substrates including $A E A$, and 4. Up-regulation of surface $C B 1$ receptors. $C B 1$ signaling is required to maintain synchronous network activity, perhaps through combined regulation of neurotransmitter release and axonal excitability. 
Figure 1

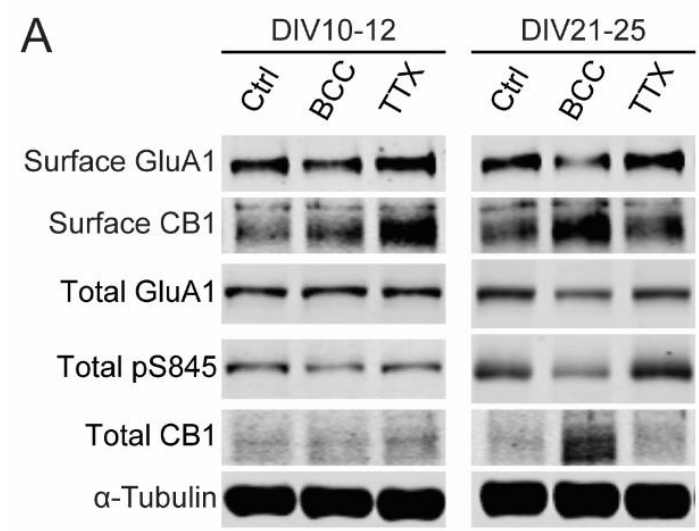

D

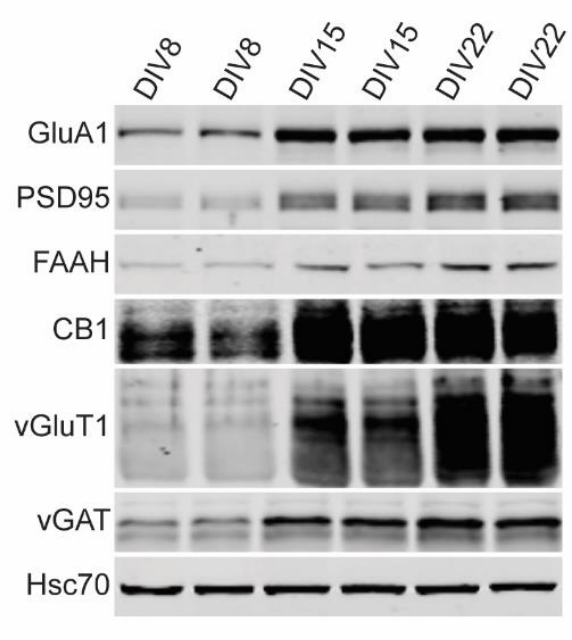

$E$
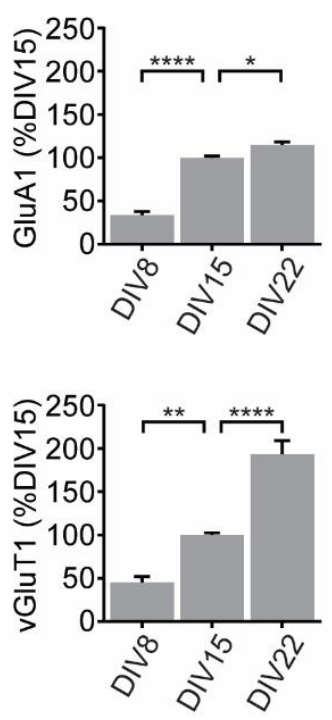

B
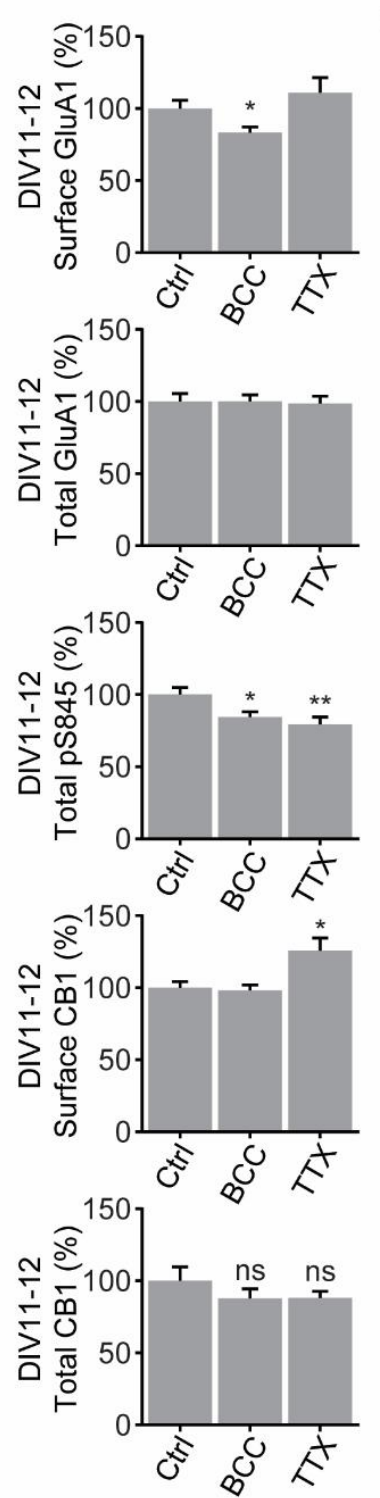

C
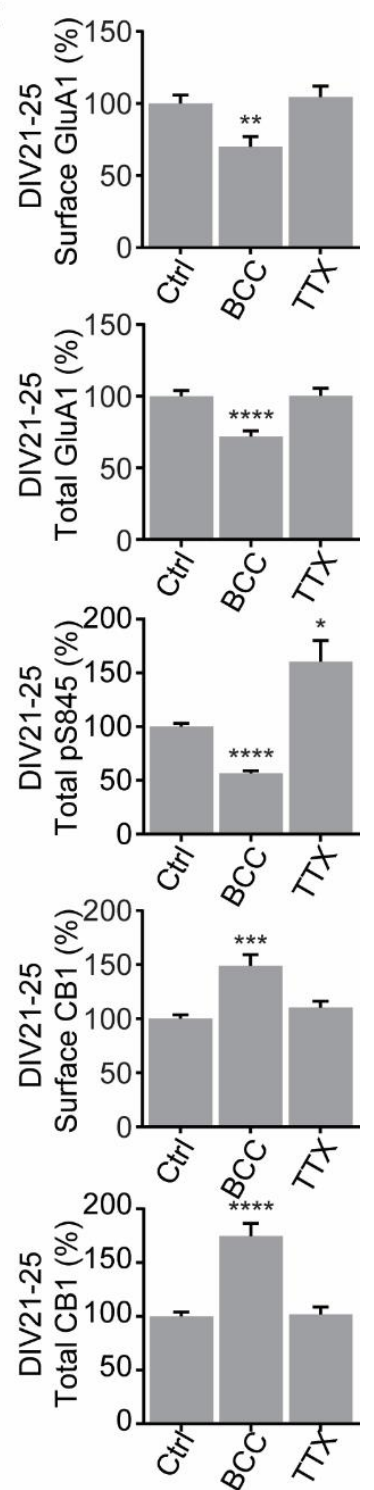
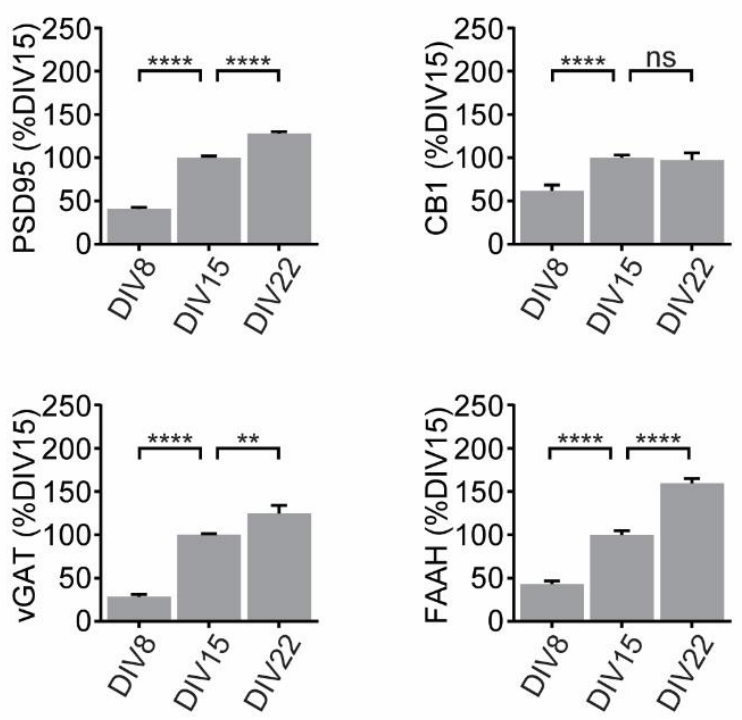
Figure 2

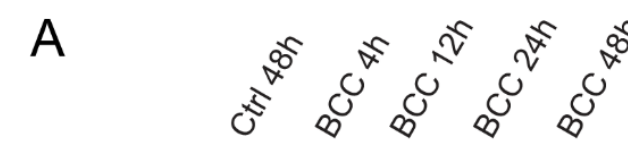

Total GluA1

Total pS845

Total CB1

Total FAAH

Tubulin

\section{B}
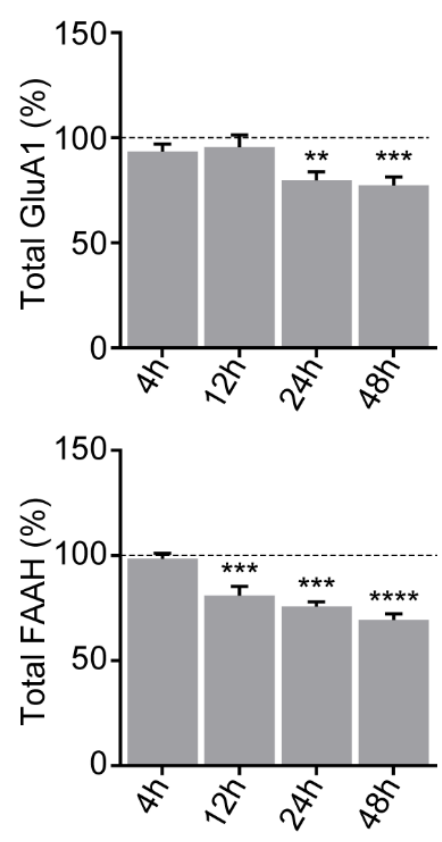

C
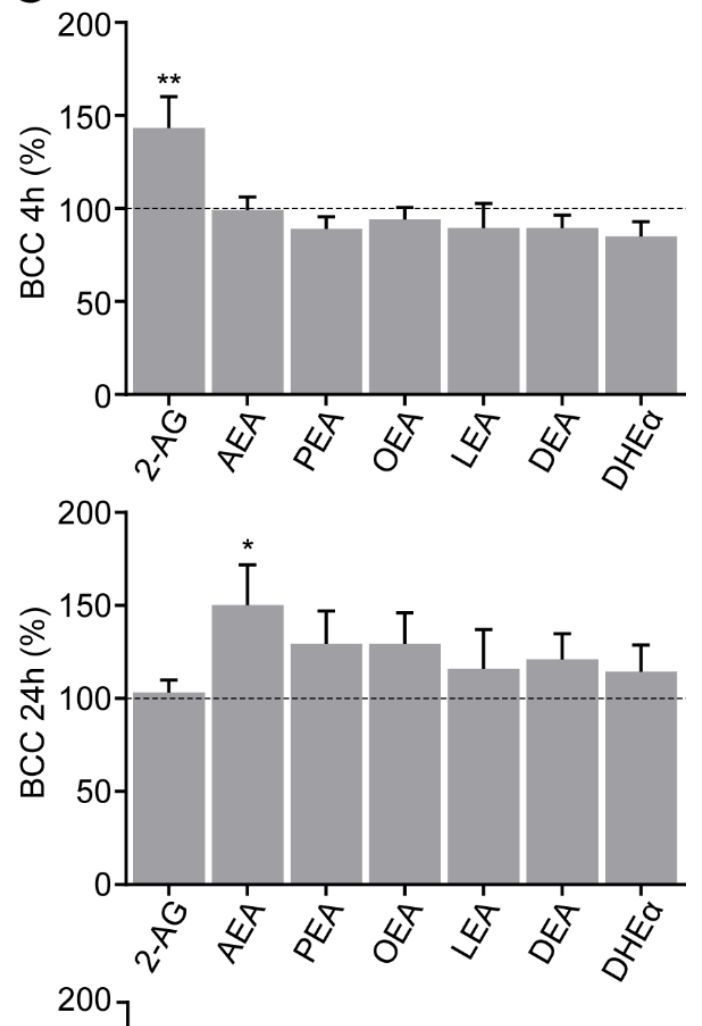
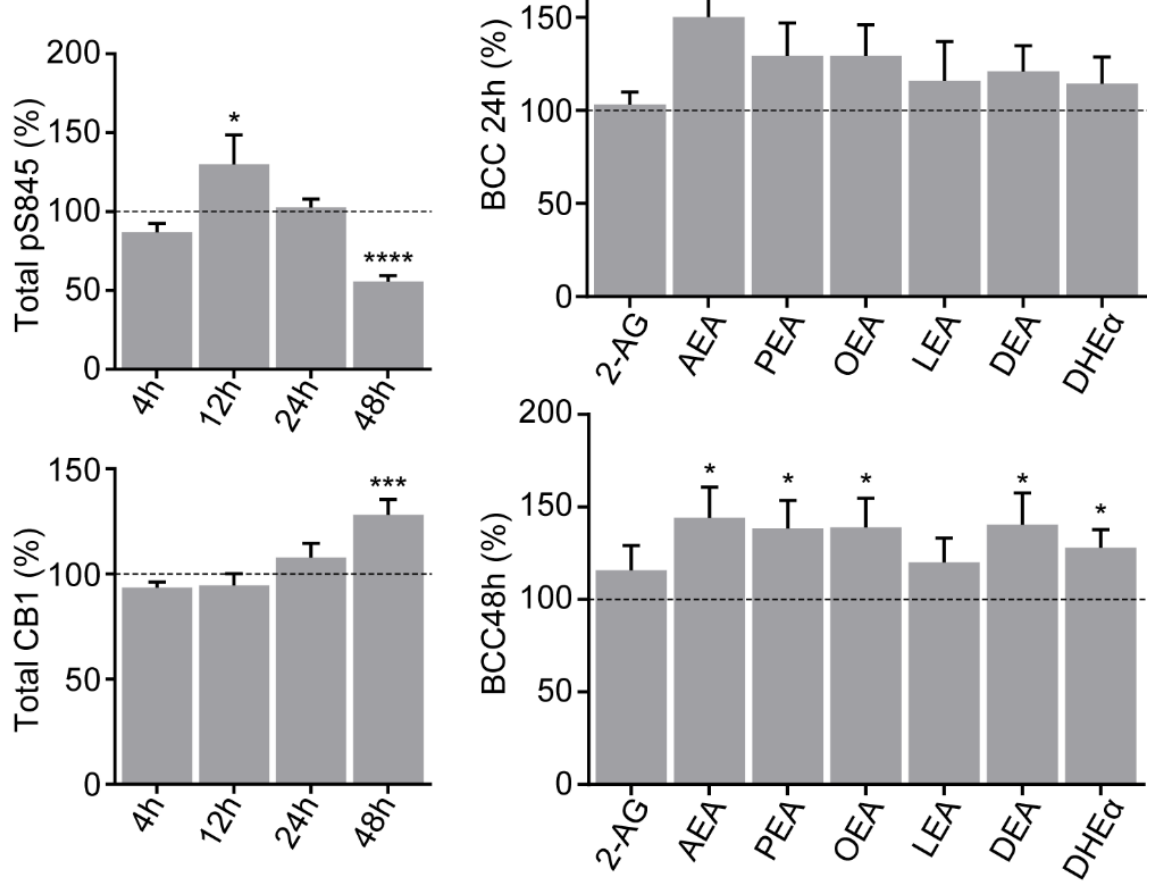


\section{Figure 3}
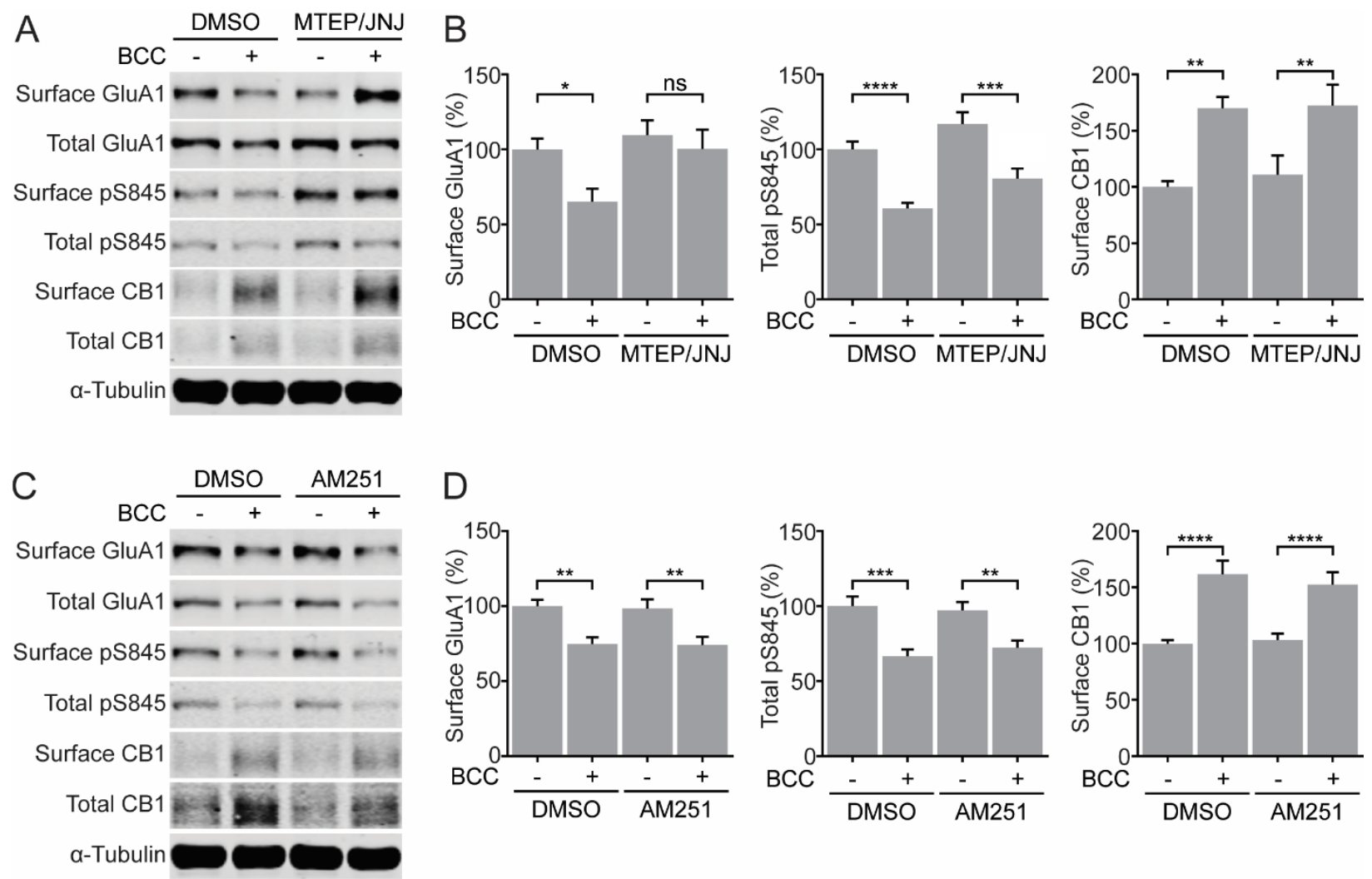
Figure 4

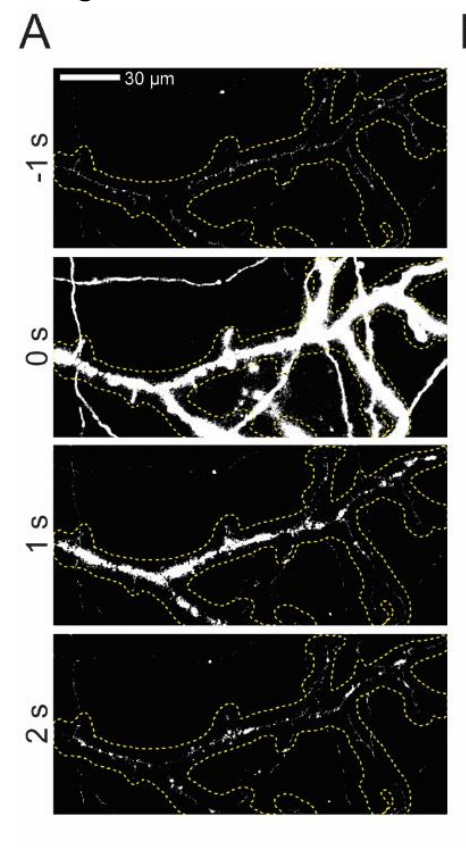

D

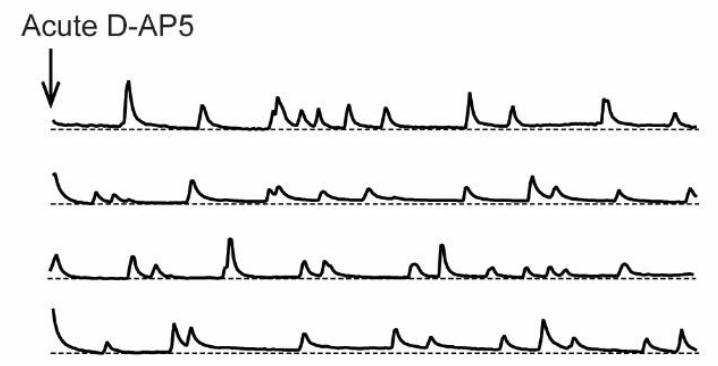

E

Acute TTX

$\downarrow$

B

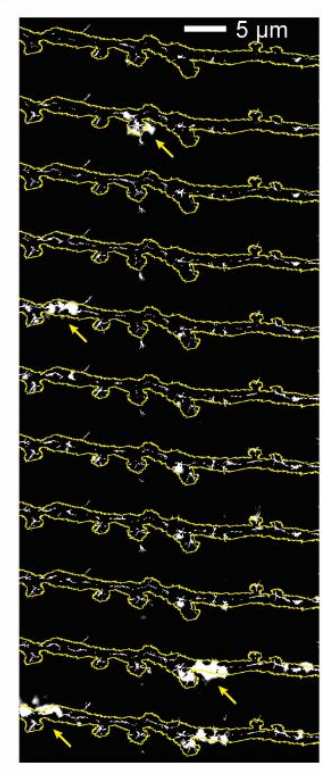

F

Acute BCC
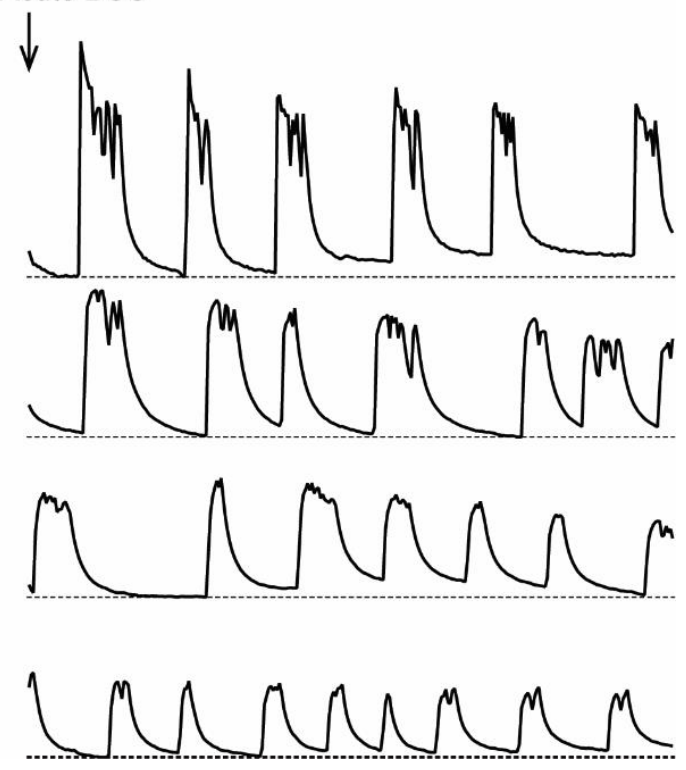


\section{Figure 5}

A

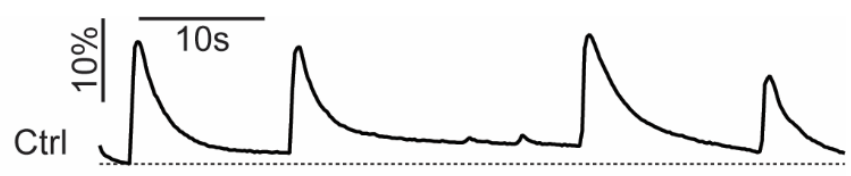

Acute
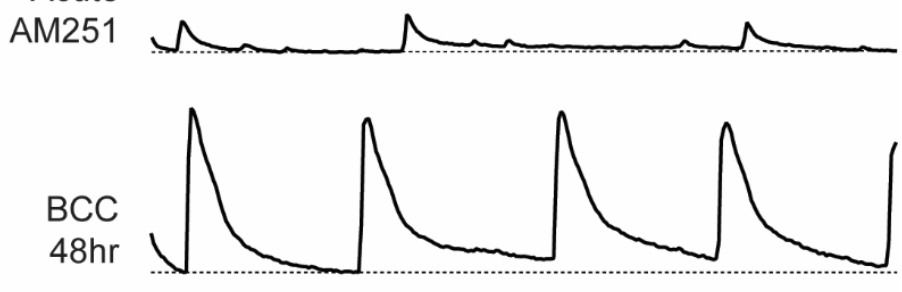

BCC 48hr+
Acute AM251

B

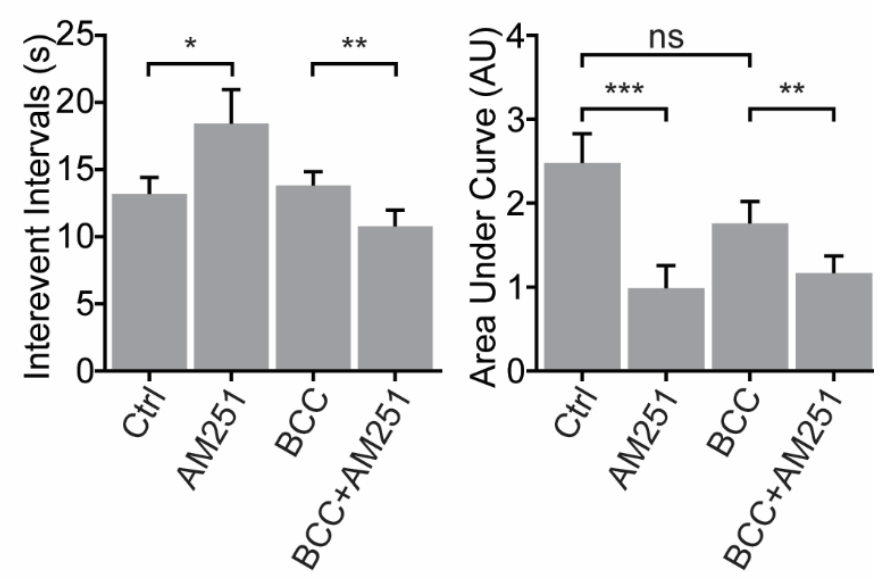


Figure 6

BASELINE

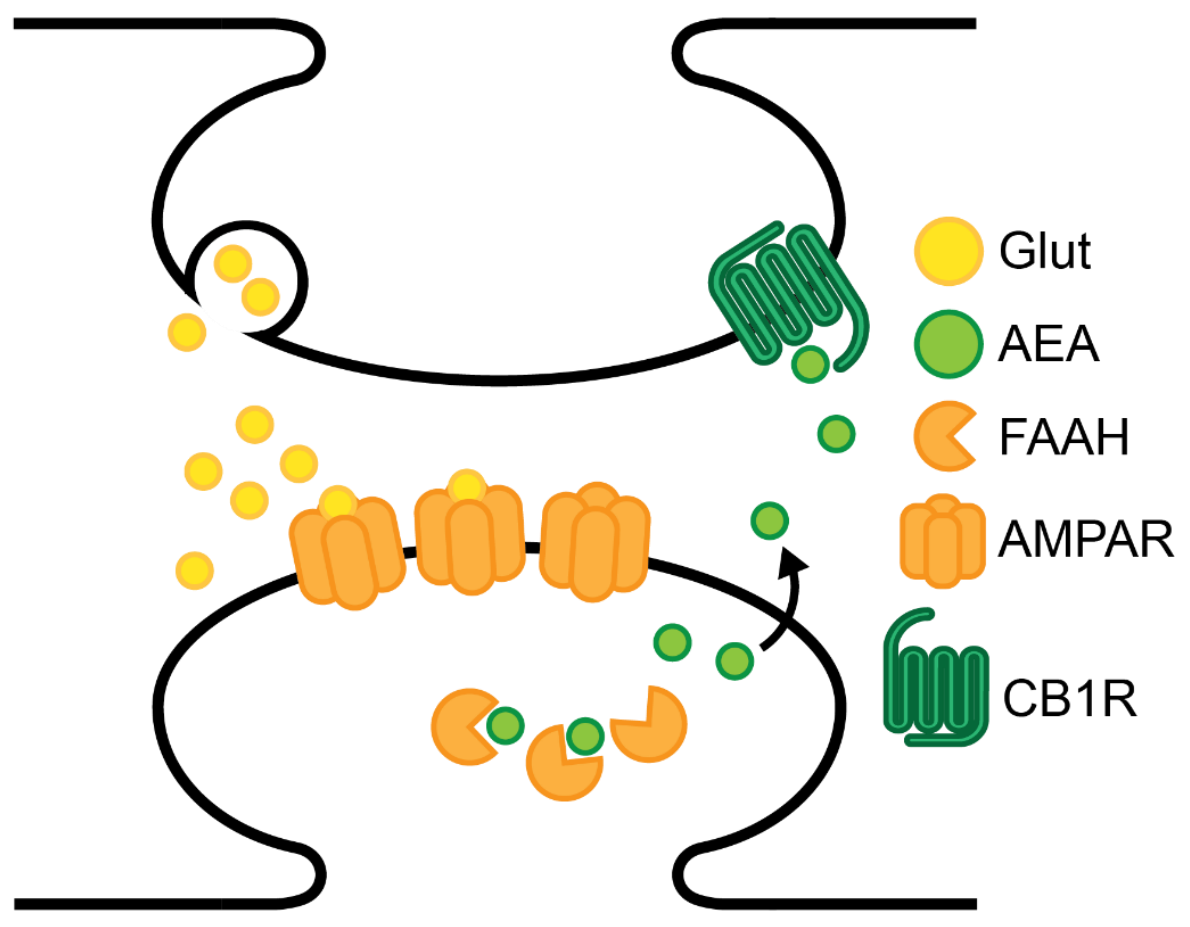

HOMEOSTATIC DOWNSCALING

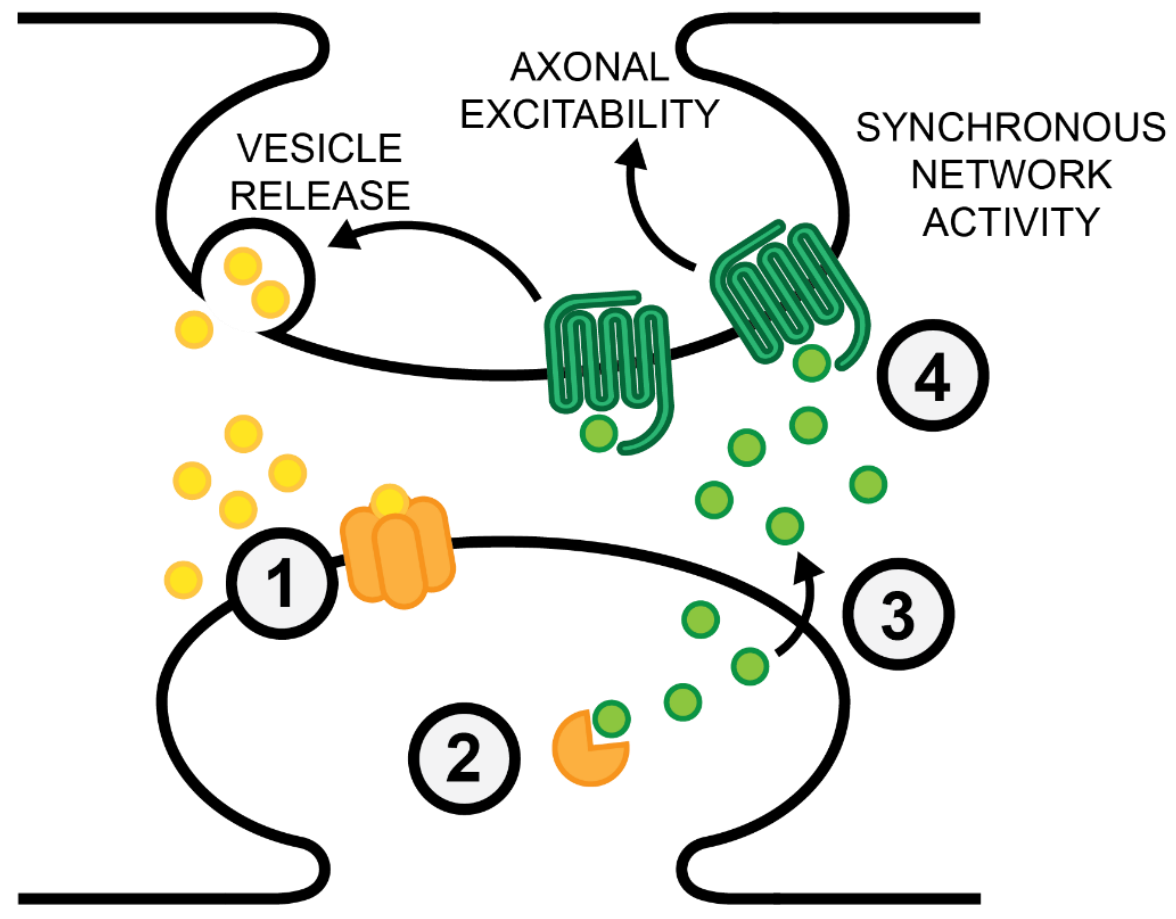

